\title{
Is there a Significant Difference in Surgery and Outcomes between Unipolar and Bipolar Hip Hemiarthroplasty? A Retrospective Study of a Single Institution in Singapore
}

\author{
WL Loo, MMed Orth, sY Loh, FRCS (Ed), HC Lee, FRCS (Ed) \\ Department of Orthopaedic Surgery, Changi General Hospital, Singapore
}

\begin{abstract}
Objective: The aim was to compare outcomes differences between unipolar and bipolar hip hemiarthroplasty in one institution for the local geriatric population. Methodology: A retrospective review of 291 patients aged 70 and above who underwent hip hemiarthroplasty for neck of femur fracture from February 2004 to November 2006 was performed. Results: Operative time was lower in the unipolar group. There was no statistically significant difference between the groups in terms of superficial wound infection, deep implant infection and hip dislocation. Patients who underwent unipolar hip hemiarthroplasty tended to be older and have a higher Charlson Comorbidity Score. Conclusions: The use of a bipolar endoprosthesis in the management of displaced femoral neck fractures in the elderly was associated with a shorter length of stay but higher hospitalisation bill, which was not statistically significant. However, the unipolar group were significantly older with a higher CCS score.
\end{abstract}

\section{INTRODUCTION}

Femoral neck fractures are a common insufficiency fracture in the elderly. Ravikumar followed 290 patients with displaced subcapital femur fractures for 13 years and reported that a higher rate of revision surgery was needed in those who underwent open reduction (as compared to those who had hemiarthroplasty or total hip arthroplasty), due to internal fixation nonunion and avascular necrosis ${ }^{1}$. Similarly, in a study of 222 patients reported by Frihagen, hip hemiarthroplasty was associated with better functional outcomes than internal fixation in the treatment of displaced fractures of the femoral neck in elderly patients ${ }^{2}$. Thus, it seems that femoral head replacement surgery is preferred in the elderly.

Some controversy surrounds which prosthesis (unipolar versus bipolar) is better in the management of these fractures. The bipolar hip prosthesis has theoretical advantages in that it is modular and has two articulating surfaces, with a reportedly lower incidence of perioperative complication of dislocation. However, it is also more expensive and technically demanding. There is little data available comparing both unipolar and bipolar hip hemiarthroplasties and the costs associated with osteoporotic hip fractures in Singapore.

We present the results of a retrospective study comparing the length of hospital stay, cost of stay, operation time and operative complications between unipolar and bipolar hip hemiarthroplasty in our local geriatric population.

\section{MATERIALS AND METHODS}

We conducted a retrospective chart review of 291 consecutive patients aged 70 and above who underwent hip hemiarthroplasty for displaced femoral neck fracture in our department from February 2004 to November 2006, a22 month period. All surgeries were either performed by or supervised by consultant surgeons. Following surgery, all patients were referred to physiotherapists and occupational therapists for rehabilitation.

Data were retrieved from clinical case notes and operative notes. Data investigated includes: age, gender, type of hip prosthesis used, average length of stay, days to surgery, and the time interval between surgery and discharge. We also reviewed the medial history of all patients and utilized the Charlson Comorbidity Score to score the patients ${ }^{3}$. (Table I). The Charlson Comorbidity Score consists of scoring 'comorbidity' points given for medical conditions such as coronary artery disease, liver disease, renal disease or metastatic tumour. The gross hospitalization cost (including ward charges, costs of surgery, implants and radiological services) was calculated by the financial department.

The patients were assigned to either the unipolar $(\mathrm{N}=177)$ or bipolar $(\mathrm{N}=114)$ groups. Patients in the unipolar group received 175 Moore (Stryker, Howmedica) and 2 Thompson implants while those in the bipolar group received cemented or uncemented implants which were chosen by the consultant surgeon. All were followed up for two years and 
Table I: Demographics, Charlson Comorbidity Score (CCS), length of stay and operating time of unipolar and bipolar groups. Days to surgery: number of days from admission to date of surgery. Surgery to discharge: numbers of days from date to surgery to date of discharge. Mann-Whitney test was used.

\begin{tabular}{|lccc|}
\hline & Unipolar & Bipolar & P value \\
\hline Age & 82.86 & 79.65 & 0.01 \\
Gender & $(+/-6.68)$ & $(+/-5.98)$ & 0.74 \\
Mean CCS & $144 \mathrm{~F}: 33 \mathrm{M}$ & $91 \mathrm{~F}: 23 \mathrm{M}$ & $(80 \%: 20 \%)$ \\
Length of stay (days) & $(81 \%: 19 \%)$ & 4.89 & 0.03 \\
Days to surgery & 5.35 & $16.85+8.29$ & 0.01 \\
Surgery to discharge (days) & $21.09+13.07$ & $5.6+5.4$ & 0.076 \\
Operating time (minutes) & $6.8+6.4$ & $11.2+5.7$ & 0.002 \\
Gross bill & $14.3+11.0$ & $93.3(+/-23.28)$ & 0.001 \\
(Singapore dollars S\$) & $63.84(+/-) 18.04$ & 12072.33 & 0.88 \\
\end{tabular}

Table II: Breakdown of operating time between unipolar, uncemented and cemented bipolar hip hemiarthroplasty using one way ANOVA.

\begin{tabular}{|lcccc|}
\hline & Unipolar & Bipolar (uncemented) & Bipolar (cemented) & P value \\
\hline No. of cases & 177 & 50 & 64 & NA \\
Operating time (minutes) & 63.8 & 86.2 & 98.8 & 0.003 \\
\hline
\end{tabular}

Table III: The Charlson Comorbidity Score is the sum of the comorbidity points ${ }^{3}$.

\begin{tabular}{|c|c|c|c|}
\hline \multicolumn{4}{|c|}{ Comorbidity Points } \\
\hline \multicolumn{4}{|c|}{$\begin{array}{l}1 \text { point each for coronary artery disease, congestive heart failure, peripheral vascular disease, cerebrovascular } \\
\text { disease, dementia, chronic pulmonary disease, connective tissue disorder, peptic ulcer disease, mild liver disease, } \\
\text { diabetes } \\
1 \text { point for every decade over } 40 \\
2 \text { points each for hemiplegia, moderate to severe renal disease, diabetes with end-organ damage, cancer } \\
3 \text { points for moderate to severe liver disease } \\
6 \text { points each for metastatic solid tumor or AIDS }\end{array}$} \\
\hline CCS score & $<3$ & $6-7$ & $>8$ \\
\hline Annual mortality rate & 0.03 & 0.27 & 0.49 \\
\hline
\end{tabular}

Table IV: Comparing hip dislocation, superficial wound infection and implant infection between unipolar and bipolar groups. Fisher's exact test was used.

\begin{tabular}{|lccc|}
\hline & Unipolar (177) & Bipolar (114) & P value \\
\hline Pneumonia & 7 & 8 & 0.28 \\
Urinary tract infection & 33 & 16 & 0.33 \\
Acute myocardial infarction & 5 & 0 & 0.16 \\
Cerebrovascular accident & 2 & 0 & 0.52 \\
Superficial wound infection & 7 & 5 & 0.99 \\
Deep wound infection & 4 & 0 & 0.15 \\
Dislocation & 5 & 2 & 0.70 \\
\hline
\end{tabular}

Table V: Cumulative mortality of patients at 30 days, 1 year and 2 year post surgery.

\begin{tabular}{|lrcc|}
\hline Cumulative & Unipolar & Bipolar & P value \\
\hline 30 day & $5(3 \%)$ & $2(2 \%)$ & 0.70 \\
1 year & $15(8 \%)$ & $5(4 \%)$ & 0.23 \\
2 year & $23(13 \%)$ & $7(6 \%)$ & 0.07 \\
\hline
\end{tabular}


postoperative complications such as acute myocardial infarction, stroke, urinary tract infection, pneumonia, superficial wound infection, deep implant infection and hip dislocation were recorded. Mortality was recorded from the computerized hospital records.

Statistical analysis was performed using SPSS for Windows (version 11.0; SPSS, Chicago, Illinois). Univariate analysis was performed with Chi-square or the Fisher's exact test for comparison of proportions between two categorical data values. The Mann-Whitney U test was used to compare the non-parametric data between two independent samples. A p value $<0.05$ was considered significant.

\section{RESULTS}

The demographics of the two groups are shown in Table II. The patients who underwent unipolar hip hemiarthroplasty were older $(82.9$ years vs. 79.7 years, $\mathrm{p}<0.01))$ and had a higher Charlson Comorbidity Score compared to those who had bipolar hip hemiarthroplasty ( 5.35 vs. $4.98, \mathrm{p}=0.03$ ). The mean hospitalization bill for both unipolar and bipolar groups was S\$11983.53 ( \pm 5592.72) and S\$12072.33 ( \pm 4000.91) respectively, a difference that was not statistically significant. All patients were followed up for 24 months.

The unipolar group had a longer average length of stay (21.0 vs. 16.8 days, $\mathrm{p}=0.01$ ) and lower mean operation time of 63.5 min compared to the bipolar group (uncemented $=86.2$ vs. cemented $=98.8 \mathrm{~min}, \mathrm{p}<0.001$ ) (Table III). The unipolar group underwent surgery on average 6.8 days after admission, compared to 5.6 days in the bipolar group ( $p=0.076$, not statistically significant). However, there was a statistical significance between the 2 groups in terms of days to discharge after surgery. The patients in the unipolar group were discharged 14.3 days on average after surgery compared to 11.2 days in the bipolar group $(\mathrm{p}=0.002)$.

In the unipolar group, there were 7 cases of superficial wound infection and 4 cases of deep implant infection. This is in contrast to 5 cases of superficial wound infection and no implant infection in the bipolar group. In terms of hip dislocation, there were 5 cases and 2 cases in the unipolar and bipolar groups respectively. Thus, there is no statistical difference between the 2 groups in term of superficial wound infection, deep implant infection or dislocation (Table IV).

No statistical difference was noted between the 2 groups in terms of postoperative morbidity such as pneumonia, urinary tract infection, myocardial infarction or stroke. In terms of 30 day mortality and mortality at 1 year, both groups were similar. (Table V). The unipolar group had a mortality rate of $3 \%$ vs. $2 \%$ (bipolar group) at 30 days post operation. At 1 year, the mortality rates were $8 \%$ vs. $4 \%$ for the unipolar and bipolar groups respectively. At 2 year, they were $13 \%$ vs. $6 \%$ respectively.
There was one case of aseptic loosening and another case of acetabular protrusion in the unipolar group.

\section{DISCUSSION}

The incidence of hip fracture in Singapore is rising. The Asian Osteoporosis Study reported a specific incidence rate of 164 and 442 per 100000 for men and women respectively, ages 50 years and above, and concluded that hip fracture will prove to be a major public health challenge ${ }^{4}$. Wong et al reported in 2002 that the average cost incurred for managing osteoporotic hip fractures was $\$ 7367$ and the average government subsidy amounted to $82 \%{ }^{5}$. The difference in average cost between the Wong study cohort and our patient group can be explained by two factors. Firstly, 58\% of his cohort consisted of intertrochanteric fractures that involved head preserving surgical stabilization. The implants used in the Wong study were less expensive than a hip prosthesis. Second, cost of hospital stay has increased over the years.

In an observational study published by Pasco, it was noted that the management of hip fractures continued to generate significant costs throughout the year following patient discharge ${ }^{6}$. The prevailing numbers of hip fractures at present and the expected rise in number in the future due to Singapore's aging population indicates that the control of costs of care for femoral neck fractures is a serious challenge for clinicians and healthcare administrators alike. Orthopaedics surgeons must choose the optimal procedure and implant for each patient, bearing in mind costs, premorbid ambulatory status, rehabilitative potential, quality of life, and life expectancy. The choice of unipolar or bipolar hip prosthesis should therefore be tailored to the needs of the patient and the availability of financial resources.

The development of the bipolar hemiarthroplasty was based in part on clinical experience with the unipolar model. Austin Moore developed the stainless steel monopolar arthroplasty in 1942 and in 1952 Thompson introduced a similar implant made of cobalt chrome alloy. Both prostheses are still in common use today.

Modularity of the bipolar implant ensures a better fit in leg length and femur size compared to "two sizes fit all" in the unipolar model. Bipolar design also dissipates joint forces through the inner bearing surfaces thereby decreasing the rate of superior acetabular erosion and the incidence of pain? In addition, the combined arc of motion of the bipolar implant reduces the incidence of dislocation. Lastly, the bipolar choice allows for an easier conversion to a total hip arthroplasty as the femoral component is then already in place.

Calder compared the unipolar and bipolar prosthesis for displaced intracapsular fracture in octogenarians and the results did not favour the use of the more expensive bipolar 
in patients more than 80 years old; further, there was no statistical difference between the rate of complications in the two groups two years after operation ${ }^{8}$. The degree of return to the pre-injury state was also significantly greater when using the unipolar prosthesis. Other studies suggest the use of unipolar prosthesis is advised in elderly patients due to the low demand on the implant, and that there is no difference in functional hip scores between the two prosthesis $9,1011,12$. Owing to the limited life expectancy of elderly patients, the mortality rate associated with hip fracture as well as cost of the bipolar implant is skewed depending on which outcome measurement was used. The mortality rate in the present study for all patients is $6.8 \%$ and this has not changed significantly in the past 10 years when compared with previous local studies ${ }^{13,14,15}$. Nather reported $15 \%$ mortality at one year for 110 patients who underwent hip hemiarthroplasty for displaced femoral neck fractures ${ }^{14}$. Lee et al followed up a group of 70 patients with a hip fracture for one year and reported $25.4 \%$ mortality for surgical patients.

The overall hip dislocation, superficial wound infection and implant infection rates in our study are $2 \%, 4 \%$ and $1 \%$ respectively, results that are comparable with other published studies ${ }^{16,17}$. Varley reviewed 133 published articles and concluded that the overall dislocation rate for all types of hemiarthroplasty was 791/23,107 (3.4\%) ${ }^{16}$. Goldhill followed 247 patients who underwent bipolar hip hemiarthroplasty and reported that 2 patients $(0.9 \%)$ had postoperative dislocations ${ }^{17}$. The overall wound infection and deep infection rate in the Goldhill study were 3.2 and $0.9 \%$ respectively. Our results showed no difference in the rates of these complications between the unipolar and bipolar groups.

There are several limitations in our study including the facts that this was a retrospective study and there was no algorithm used in choosing an implant (unipolar or bipolar hip hemiarthroplasty) for each patient. The 24-month followup period is also short. The difference in complication rates of aseptic loosening and acetabular protrusio between these two groups is small as these failure mechanisms are likely to increase with longer term follow-up.

\section{CONCLUSION}

Hemiarthroplasty for displaced femoral neck fractures in our hospital yields comparable results to international studies in terms of morbidity and mortality. The costs for the hospital stay were comparable for the unipolar and bipolar groups. The use of a bipolar endoprosthesis in the management of displaced femoral neck fractures in the elderly was associated with a longer duration of operation but a shorter length of stay. Possible explanations for this include preselection of younger and healthier patients with no significant comorbidities for bipolar hip hemiarthroplasty. For instance, the patients in the unipolar group were older, and had a higher Charlson Comorbidity Score, requiring as would be expected, a longer duration of stay before transfer to a step-down medical facility. 


\section{REFERENCES}

1. Ravikumar KJ, Marsh G. Internal fixation versus hemiarthroplasty versus total hip arthroplasty for displaced subcapital fractures of femur — 13 year results of a prospective randomised study. Injury 2000; 3(10): 793-7.

2. Frihagen F, Nordsletten L, Madsen JE. Hemiarthroplasty or internal fixation for intracapsular displaced femoral neck fractures: randomized controlled trial. BMJ 2007; 335(7632): 1251-4.

3. Charlson M, Szatrowski TP, Peterson J, Gold J. Validation of a combined comorbidity index. J Clin Epidemiol 1994; 47: 1245-51.

4. Lau EM, Lee JK, Suriwongpaisal P, Saw SM, Das De S, Khir A, Sambrook P et al. The incidence of hip fracture in four Asian countries: the Asian Osteoporosis Study (AOS). Osteoporos Int 2001; 12(3): 239-43.

5. Wong MK, Arjandas, Ching LK, Lim SL, Lo NN. Osteoporotic hip fractures in Singapore - costs and patient's outcome. Ann Acad Med Singapore 2002; 31(1): 3-7.

6. Pasco JA, Sanders KM, Hoekstra FM, Henry MJ, Nicholson GC, Kotowicz MA. The human cost of fracture. Osteoporos Int 2005; 16(12): 2046-52.

7. Lestrange NR. Bipolar arthroplasty for 496 hip fractures. Clin Orthop Relat Res 1990; (251): 7-19.

8. Calder SJ, Anderson GH, Jagger C, Harper WM, Gregg PJ. Unipolar or Bipolar Prosthesis for displaced intracapsular hip fracture in octogenarians: a randomized prospective study. J Bone Joint Surg [Br] 1996; 78(3): 391-4.

9. Cornell CN, Levine D, O’Doherty J, Lyden J. Unipolar versus bipolar hemiarthroplasty for the treatment of femoral neck fractures in the elderly. Clin Orthop Relat Res 1998; 67-71.

10. Gilbert MS, Capozzi J. Unipolar or bipolar prosthesis for the displaced intracapsular hip fracture? An unanswered question. Clin Orthop Relat Res 1998; (353): 81-5.

11. Ong BC, Maurer SG, Aharonoff GB, Zuckerman JD, Koval KJ. Unipolar versus bipolar hemiarthroplasty: functional outcome after femoral neck fracture at a minimum of thirty six months of follow up. J Orthop Trauma 2002; 16(5): 317-22.

12. Raia FJ, Chapman CB, Herrera MF, Schweppe MW, Michelsen CB, Rosenwasser MP. Unipolar or bipolar hemiarthroplasty for femoral neck fractures in the elderly? Clin Orthop Relat Res 2003; 414: 259-65.

13. Lin KH, Lim YW, Wu YJ, Lam KS. Mortality after proximal hip fracture in the Singapore population. Hip International 2005; 15: $166-70$.

14. Nather A, Seow CS, Iau P, Chan A. Morbidity and mortality for elderly patients with fractured neck of femur treated by hemiarthroplasty. Injury 1995; 26(3): 187-90.

15. Lee AY, Chua BS, Howe TS. One year outcome of hip fracture patients admitted to a Singapore hospital: quality of life posttreatment. Singapore Med J 2007; 48(11): 996.

16. Varley J, Parker MJ. Stability of hip arthroplasties. Int Orthop 2004; 28(5): 274-7.

17. Goldhill VB, Lyden JP, Cornell CN, Bochner RM. Bipolar hemiarthroplasty for fracture of the femoral neck. J Orthop Trauma 1991; 5(3): 318-24. 